\title{
Successful Treatment with Lorlatinib after the Development of Alectinib-Induced Liver Damage in ALK-Positive Non-Small-Cell Lung Cancer: A Case Report
}

\author{
Go Makimoto Keita Kawakado Masamoto Nakanishi Tomoki Tamura \\ Shoichi Kuyama
}

Department of Respiratory Medicine, National Hospital Organization Iwakuni Clinical

Center, Iwakuni, Japan

\section{Keywords}

Alectinib · Lorlatinib · Non-small-cell lung cancer · Anaplastic lymphoma kinase ·

Hepatotoxicity

\section{Abstract}

Alectinib is a key drug for treating anaplastic lymphoma kinase $(A L K)$-positive non-small-cell lung cancer (NSCLC). Alectinib-induced hepatotoxicity is less common than that through other ALK inhibitors, such as crizotinib or ceritinib. Herein, we describe a case of $A L K$-positive adenocarcinoma successfully treated with lorlatinib after developing alectinib-induced hepatotoxicity. A 57-year-old Japanese man received alectinib as first-line therapy for $A L K$-positive NSCLC. After 79 days, alectinib was discontinued because of hepatotoxicity and later restarted at $150 \mathrm{mg} /$ day, inducing hepatotoxicity again after 64 days. Switching to lorlatinib treatment (continued for $>4$ months) caused no severe adverse effects. Hence, lorlatinib may be useful for patients experiencing alectinib-induced hepatotoxicity.

\section{Introduction}

In 2007, the echinoderm microtubule-associated protein-like 4 (EML4)-anaplastic lymphoma kinase (ALK) fusion gene was proven to cause non-small-cell lung cancer (NSCLC) [1]. The EML4-ALK fusion gene is present in approximately 3-5\% of NSCLC patients [2]. ALK tyrosine kinase gets automatically activated by multimerization with fusion partners, leading to cancers through the overactivation of cell proliferation signals. Hence, blocking the acti- 


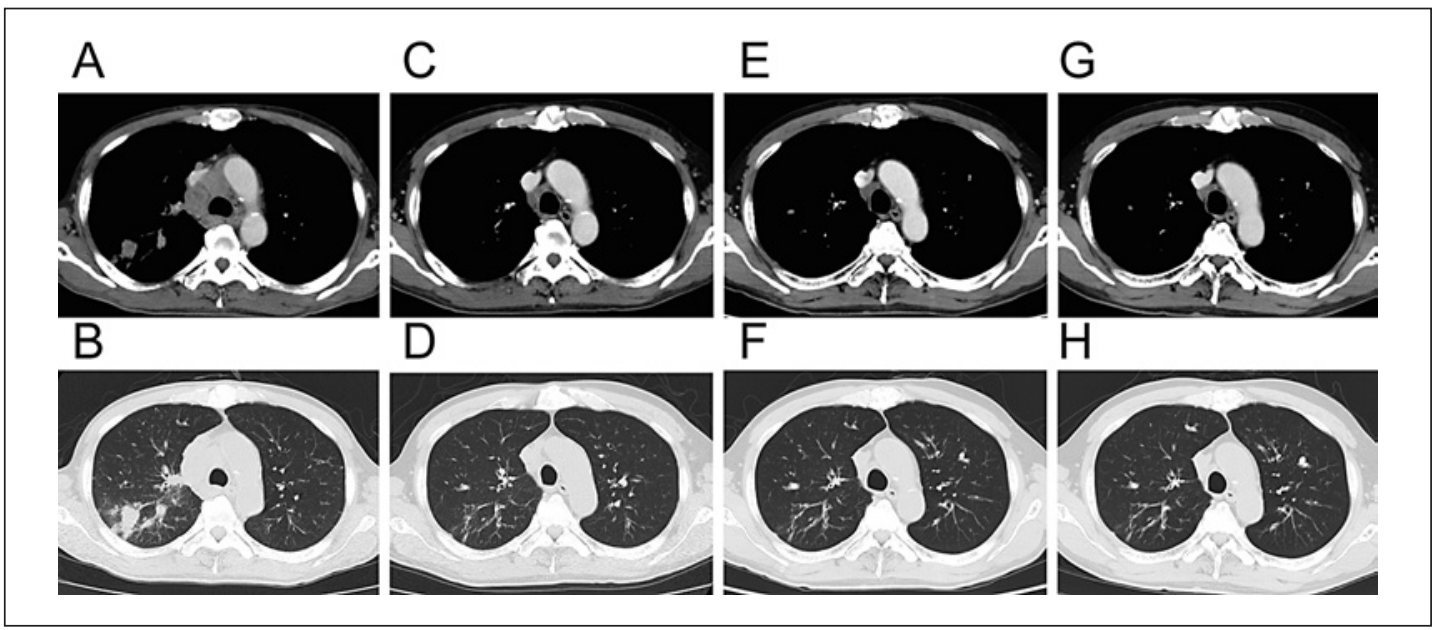

Fig. 1. Computed tomography images of the lung tumor immediately before the administration of alectinib (A, B), after initial alectinib-induced hepatotoxicity (C, D), after second-time alectinib-induced hepatotoxicity (E, F), and 4 months after the initiation of lorlatinib $(\mathbf{G}, \mathbf{H})$. The right upper primary lung tumors almost disappeared at 3 and 7 months after alectinib therapy and 4 months after lorlatinib therapy.

vation of ALK tyrosine kinase significantly inhibits cell proliferation in $A L K$ fusion genepositive cancer cells. To date, four ALK tyrosine kinase inhibitors (ALK-TKIs) have been approved: the first-generation ALK-TKI crizotinib, the second-generation ALK-TKIs alectinib and ceritinib, and the third-generation ALK-TKI lorlatinib. Notably, the second-generation ALK-TKI alectinib has shown a high objective response rate $(93.5 \%)$ and a long progressionfree survival rate in $A L K$-positive NSCLC patients (3-year progression-free survival in $62 \%$ of cases) [3]. Additionally, alectinib is associated with fewer severe adverse effects, such as diarrhea and nausea, compared to crizotinib [4]. Thus, alectinib is widely used as the first-line treatment in $A L K$-positive lung cancer patients.

Hepatotoxicity is one of the common adverse effects of ALK-TKIs, such as crizotinib or ceritinib $[4,5]$. Alectinib exhibits a lower frequency of hepatotoxicity than crizotinib or ceritinib [6,7], accounting for fewer reports of alectinib-induced severe liver damage [8]. Although several cases have been reported where crizotinib-induced hepatotoxicity was treated [9], there are no established strategies to overcome the hepatotoxicity of ALK-TKIs, especially alectinib.

The third-generation ALK-TKI lorlatinib was developed as a selective and brain-penetrating ALK inhibitor. In a phase 1 single-arm, first-in-human, dose-escalation study, lorlatinib demonstrated an objective response in $19 / 41$ patients (46\%; 95\% confidence interval [CI] 31-63) who had received two or more ALK-TKIs [10]. A subsequent global phase 2 study was conducted to evaluate the efficacy of lorlatinib. During this trial, $A L K$-positive NSCLC patients were enrolled in different expansion cohorts. In the ALK-TKI treatmentnaive patients (EXP1 group, $n=30$ ), the objective response rate was $27 / 30(90.0 \%$; $95 \%$ CI 73.5-97.9) [11]. The other expansion cohorts consisting of patients resistant to other ALK-TKIs also demonstrated a favorable objective response rate (EXP2-5 group, resistant to other ALK-TKIs; the objective response rate was 93/198 [47.0\%; 95\% CI 39.9-54.2]). Therefore, lorlatinib was approved in 2018 for ALK-TKI-resistant or -intolerant ALKpositive NSCLC.

Herein, we report a rare case of a patient with $A L K$-positive adenocarcinoma who was successfully treated with lorlatinib after the development of alectinib-induced liver damage. 
Fig. 2. Change in the serum levels of aspartate aminotransferase (AST), alanine aminotransferase (ALT), total bilirubin (T-Bil), and tumor markers over the clinical course of the disease. KL-6, Krebs von den Lungen-6; SNMC, glycyrrhizin; UDCA, ursodeoxycholic acid.

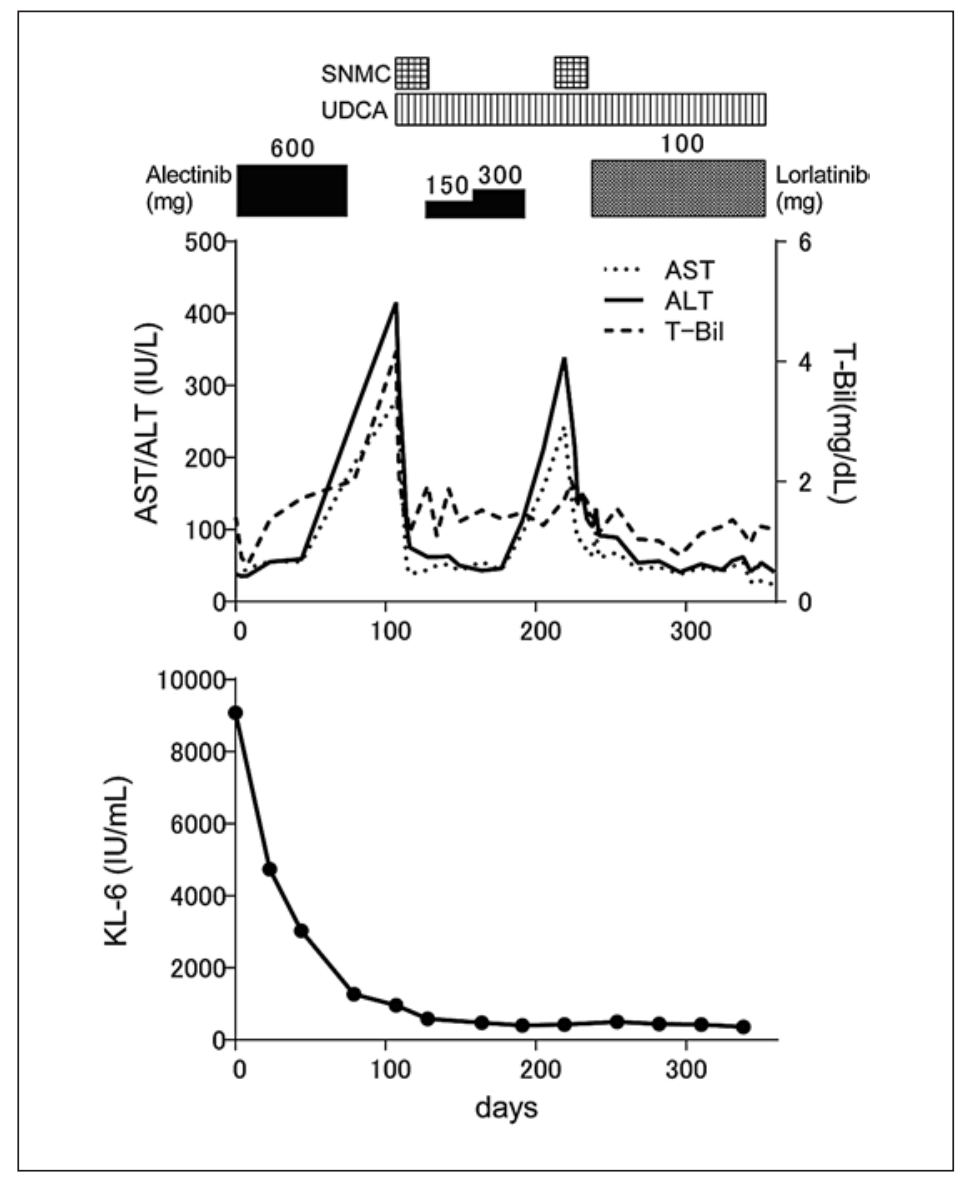

\section{Case Report}

A 57-year-old man was referred to our hospital because of multiple lung tumors and cervical lymph node swelling and was diagnosed with advanced lung adenocarcinoma (cT1bN3M1c stage IVB) (Fig. 1A, B). Although he was an ex-smoker with 23 pack-years, the tumor was ALK-positive on immunohistochemistry. Moreover, we submitted fresh frozen tissue specimens to the Cancer Genome Screening Project for Individualized Medicine in Japan (SCRUM-JAPAN), following which an EML4-ALK fusion was detected using the Oncomine ${ }^{\circledR}$ Comprehensive Assay (version 3). Alectinib (600 mg/day) was then administered as first-line therapy. After the initiation of alectinib, the tumor and lymph node swelling decreased rapidly (Fig. 1C, D). However, on day 79 of treatment, the serum levels of aspartate aminotransferase (AST) and alanine aminotransferase (ALT) increased to grade 3, according to the Common Terminology Criteria for Adverse Events (CTCAE) v5.0. Alectinib was discontinued, but the levels of serum AST and ALT remained elevated. Consequently, he was readmitted to our hospital for treatment of alectinib-induced hepatotoxicity on day 107. Abdominal computed tomography and ultrasonography revealed no significant abnormality of the liver. Hepatitis A, B, and C were also excluded. Antibodies (IgM) to cytomegalovirus and EpsteinBarr virus were also negative. The pattern of liver injury suggested that it was a cholestatictype injury, according to both the Digestive Disease Week Japan 2004 scale and the $R$ value $[12,13]$. Therefore, it was determined that the patient had alectinib-induced hepatotoxicity. He was treated with oral ursodeoxycholic acid (UDCA) $600 \mathrm{mg} /$ day and intravenous glycyr- 
rhizin (SNMC). The hepatotoxicity gradually improved to grade 1 (Fig. 2). We then tried to rechallenge the patient with alectinib at a reduced dose of $150 \mathrm{mg} /$ day on day 128 . After 3 weeks, the dose was increased to $300 \mathrm{mg} /$ day without severe hepatotoxicity and tumor progression (Fig. 1E, F). However, on day 191, the serum AST and ALT levels increased to CTCAE grade 2 again, necessitating the discontinuation of alectinib treatment. On day 219, the serum AST/ALT level further increased to CTCAE grade 3; therefore, the patient was readmitted for treatment with intravenous SNMC. Subsequently, the hepatotoxicity gradually improved to grade 1 . Since alectinib was considered intolerable because of hepatotoxicity, we switched to lorlatinib treatment on day 235. Thereafter, the serum AST/ALT level did not increase to grade 2 or more, and the tumor continued to respond to lorlatinib for over 4 months without any severe adverse effects (Fig. 1G, H, 2).

\section{Discussion}

We successfully treated our patient with lorlatinib after development of alectinib-induced liver damage. Among the ALK-TKIs, alectinib is recommended as the first-line treatment for $A L K$ positive NSCLC because of its safety and efficacy in the first-line setting [4, 5]. Although druginduced hepatotoxicity is more common with crizotinib or ceritinib than alectinib [6,7], approximately $1-5 \%$ of patients experience alectinib-induced liver damage [4]. Nevertheless, there have been no previous reports describing a protocol to overcome alectinib-induced liver damage.

Regarding overcoming ALK-TKI-induced hepatotoxicity, several cases have been reported, especially for crizotinib $[9,14]$. In one such report, the majority of the liver damage was of the hepatocellular type, and crizotinib was successfully resumed later at a lower dose (100 mg/day) [14]. However, in the present case, the liver damage pattern was cholestatic type according to both the Digestive Disease Week Japan 2004 scale and the $R$ value, and rechallenge with a lower dose of alectinib (a quarter of the full dose) was unsuccessful. Therefore, we sought a better method to overcome liver damage.

Lorlatinib, which is a third-generation ALK-TKI, is effective for treating $A L K$-positive NSCLC in both ALK-TKI treatment-naive and ALK-TKI-pretreated patients [11]. Its pharmacokinetic property is different from that of other ALK-TKIs as it is metabolized mainly by CYP3A4 and UDP-glucuronosyltransferase (UGT1A4), whereas crizotinib, ceritinib, and alectinib are predominantly metabolized by the cytochrome P450 (CYP450) pathway [15]. In addition, lorlatinib-induced hepatotoxicity is relatively rare compared to that induced by crizotinib or ceritinib (grade 3 or higher serum AST/ALT elevation; lorlatinib, crizotinib, ceritinib: $-1 \%, 11-15 \%$, and $17-31 \%$, respectively) $[4,5,11]$. Consequently, we switched to lorlatinib and successfully continued the treatment.

In conclusion, we have described a case in whom alectinib-induced hepatotoxicity was treated successfully with lorlatinib. Although there are no established protocols to overcome ALK-TKI-related liver damage, ALK-TKIs can achieve better clinical outcomes compared with conventional cytotoxic chemotherapy. Therefore, a more data-driven approach for appropriate protocols to manage molecular targeted drug-induced hepatotoxicity is needed for patients suffering from drug-induced adverse effects.

\section{Acknowledgement}

We would like to thank the patient for providing consent to publish clinical information and data. We also thank Editage (www.editage.jp) for English language editing of the manuscript.

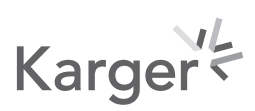




\section{Case Reports in Oncology}

\begin{tabular}{l|l}
\hline Case Rep Oncol 2021;14:197-201 \\
\hline DOI: 10.1159/000513624 & $\begin{array}{l}\text { @ 2021 The Author(s). Published by S. Karger AG, Basel } \\
\text { www.karger.com/cro }\end{array}$ \\
\hline
\end{tabular}

Makimoto et al.: Treatment Using Lorlatinib for Alectinib-Induced Liver Damage

\section{Statement of Ethics}

This case report was prepared and completed following the guidelines reported in the revised Helsinki Declaration of 2013. Written informed consent was obtained from the patient for the publication of the case report and accompanying images.

\section{Conflict of Interest Statement}

The authors have no conflicts of interest to declare.

\section{Funding Sources}

This research did not receive any specific grants from funding agencies in the public, commercial, or not-for-profit sectors.

\section{Author Contributions}

All of the authors contributed to the treatment of the patient.

\section{References}

1 Soda M, Choi YL, Enomoto M, Takada S, Yamashita Y, Ishikawa S, et al. Identification of the transforming EML4-ALK fusion gene in non-small-cell lung cancer. Nature. 2007 Aug;448(7153):561-6.

2 Shaw AT, Yeap BY, Mino-Kenudson M, Digumarthy SR, Costa DB, Heist RS, et al. Clinical features and outcome of patients with non-small-cell lung cancer who harbor EML4-ALK. J Clin Oncol. 2009 Sep;27(26):4247-53.

3 Tamura T, Kiura K, Seto T, Nakagawa K, Maemondo M, Inoue A, et al. Three-year follow-up of an alectinib phase I/II study in ALK-positive non-small-cell lung cancer: AF-001JP. J Clin Oncol. 2017 May;35(14):1515-21.

4 Camidge DR, Dziadziuszko R, Peters S, Mok T, Noe J, Nowicka M, et al. Updated efficacy and safety data and impact of the EML4-ALK fusion variant on the efficacy of alectinib in untreated ALK-positive advanced nonsmall cell lung cancer in the global phase III ALEX study. J Thorac Oncol. 2019 Jul;14(7):1233-43.

5 Soria JC, Tan DSW, Chiari R, Wu YL, Paz-Ares L, Wolf J, et al. First-line ceritinib versus platinum-based chemotherapy in advanced ALK-rearranged non-small-cell lung cancer (ASCEND-4): a randomised, open-label, phase 3 study. Lancet. 2017 Mar;389(10072):917-29.

6 Liu B, Yuan M, Sun Y, Cheng Z, Zhang Z, Hou S, et al. Incidence and risk of hepatic toxicities associated with anaplastic lymphoma kinase inhibitors in the treatment of non-small-cell lung cancer: a systematic review and meta-analysis. Oncotarget. $2018 \mathrm{Feb}$; 9(10):9480-8.

7 Li J, Yuan Z, Wang Q, Fan W, Zhang G. Meta-analysis of overall incidence and risk of ALK inhibitors-induced liver toxicities in advanced non-small-cell lung cancer. Medicine (Baltimore). 2019 Jan;98(1):e13726.

8 Zhu VW, Lu Y, Ou SI. Severe acute hepatitis in a patient receiving alectinib for ALK-positive non-small-cell lung cancer: histologic analysis. Clin Lung Cancer. 2019 Jan;20(1):e77-80.

9 Kreitman K, Nair SP, Kothadia JP. Successful treatment of crizotinib-induced fulminant liver failure: a case report and review of literature. Case Reports Hepatol. 2020 Mar;2020:8247960.

10 Shaw AT, Felip E, Bauer TM, Besse B, Navarro A, Postel-Vinay S, et al. Lorlatinib in non-small-cell lung cancer with ALK or ROS1 rearrangement: an international, multicentre, open-label, single-arm first-in-man phase 1 trial. Lancet Oncol. 2017 Dec;18(12):1590-9.

11 Solomon BJ, Besse B, Bauer TM, Felip E, Soo RA, Camidge DR, et al. Lorlatinib in patients with ALK-positive non-small-cell lung cancer: results from a global phase 2 study. Lancet Oncol. 2018 Dec;19(12):1654-67.

12 Chalasani NP, Hayashi PH, Bonkovsky HL, Navarro VJ, Lee WM, Fontana RJ. ACG Clinical Guideline: the diagnosis and management of idiosyncratic drug-induced liver injury. Am J Gastroenterol. 2014 Jul;109(7):950-67.

13 Hanatani T, Sai K, Tohkin M, Segawa K, Kimura M, Hori K, et al. A detection algorithm for drug-induced liver injury in medical information databases using the Japanese diagnostic scale and its comparison with the Council for International Organizations of Medical Sciences/the Roussel Uclaf Causality Assessment Method scale. Pharmacoepidemiol Drug Saf. 2014 Sep;23(9):984-8.

14 Ota T, Masuda N, Matsui K, Yamada T, Tanaka N, Fujimoto S, et al. Successful desensitization with crizotinib after crizotinib-induced liver injury in ROS1-rearranged lung adenocarcinoma. Intern Med. 2019 Sep;58(18):2651-5.

15 Zhao D, Chen J, Chu M, Long X, Wang J. Pharmacokinetic-based drug-drug interactions with anaplastic lymphoma kinase inhibitors: a review. Drug Des Devel Ther. 2020;14:1663-81.

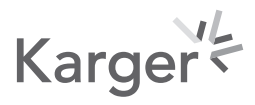

